\title{
INFLUÊNCIA DA PODA DE FORMAÇÃO SOBRE O CICLO FENOLÓGICO DE PIMENTEIRAS EM VASO
}

\author{
Renata Ranielly Pedroza Cruz ${ }^{1}$, Ana Carolina Bezerra², Amadeu Pimentel Travassos ${ }^{3}$, Eliane Nunes da \\ Silva $^{3}$, Wellington Souto Ribeiro ${ }^{4}$, Fernando Luiz Finger ${ }^{5}$
}

\begin{abstract}
RESUMO - Este estudo teve como objetivo determinar o efeito da poda de formação sobre o ciclo fenológico de dois genótipos de pimenteira para fins ornamentais: Pirâmide Ornamental (Capsicum frutescens) e Biquinho (Capsicum chinense). A primeira poda foi realizada quando as plantas apresentavam quatro a cinco pares de folhas verdadeiras. Os cortes foram realizados logo após o primeiro par de folhas apicais completamente expandidas. Quando as plantas iniciaram as brotações laterais e estas já estavam com mais de $5 \mathrm{~cm}$, foi realizada a segunda poda, retirando o excesso de brotações laterais e mantendo apenas aquelas mais vigorosas de forma a se obter os seguintes tratamentos: plantas com duas hastes, plantas com três hastes e plantas sem poda (controle). Durante a condução do experimento, as pimenteiras foram avaliadas quanto à precocidade, caracterizada pelo tempo compreendido entre o plantio e a comercialização. A poda retardou em 15 e 10 dias a precocidade dos genótipos Biquinho e Pirâmide Ornamental, respectivamente. No entanto, a fase de frutificação e maturação não foram alteradas, com exceção do genótipo Biquinho conduzido em duas hastes que retardou em 5 dias a fase de maturação.
\end{abstract}

Palavras chave: biquinho, ciclo fenológico, pirâmide ornamental, plantas ornamentais.

\section{INFLUENCE OF FORMATION PRUNING ON THE PHENOLOGICAL CYCLE OF ORNAMENTAL PEPPER IN POTTED}

\begin{abstract}
This study aimed to determine the effect of pruning on the phenological cycle of two pepper genotypes for ornamental purposes: Pirâmide ornamental (Capsicum frutescens) and Biquinho (Capsicum chinense). The first pruning was performed when the plants had four to five pairs of true leaves. The cuts were performed shortly after the first pair of fully expanded apical leaves. When the plants started the lateral shoots and they were already more than $5 \mathrm{~cm}$, the second pruning was done, removing the excess of lateral shoots and maintaining only those more vigorous so as to obtain the following treatments: plants with two stems, plants With three stems and plants without pruning (control). During the experiment, peppercorns were evaluated for precocity, characterized by the time between planting and commercialization. The pruning delayed in 15 and 10 days the precocity of the Biquinho and Pirâmide Ornamental genotypes, respectively. However, the fruiting and maturation phase were not altered, except for the genotype Biquinho conducted on two stems that delayed the maturation phase by 5 days.
\end{abstract}

Keywords: biquinho, ornamental plants, ornamental pyramid, phenological cycle.

\footnotetext{
${ }^{1}$ Graduanda em Agronomia da Universidade Federal da Paraíba, Campus II, Areia, renataranielly426@ gmail.com;

${ }^{2}$ Mestranda pelo Programa de Pós-Graduação em Agronomia da Universidade Federal da Paraíba, Campus II, Areia, acbezerra78@ gmail.com;

${ }^{3}$ Graduando em Agroecologia da Universidade Estadual da Paraíba, Campus II, Lagoa Seca, amadeutravassos@gmail.com; lianbela2010@gmail.com;

${ }^{4}$ Dr. em Fitotecnia, UFV. Prof. do bacharel em Agroecologia, curso técnico em Agropecuária e curso técnico em Agroindústria da Universidade Estadual da Paraíba, Campus II - Lagoa Seca, wellingtisouto@ yahoo.com.br

${ }^{5}$ PhD em Horticulture, Ohio University. Prof. da Universidade Federal de Viçosa, UFV, Viçosa, ffinger@ufv.br
} 


\section{INTRODUÇÃO}

Conceitualmente, a poda é definida como a arte de orientar as plantas, compatível com a finalidade da exploração (Nunes et al., 2010). Partindo deste pressuposto, deve-se fazer um estudo de caso, a fim de utilizar as técnicas de poda adequadas (Dalastra et al., 2009).

Em pimenteiras, a prática da poda tem sido efetuada no sentido de maximizar a produção por planta. No Ceará, a poda é efetuada duas vezes durante o ciclo da cultura de pimenta Tabasco, sendo uma no viveiro e a outra no campo. No viveiro, a poda é realizada a partir dos dois dias antes do transplante, deixando quatro folhas definitivas. A segunda poda é realizada 25 dias após o transplante e tem a finalidade de estimular a formação de novos ramos, inclusive os frutíferos que estão relacionados com a produção por planta. A prática contribui para a arquitetura da planta adulta em forma de taça, possibilitando uma melhor distribuição dos frutos (Crisóstomo et al., 2006). No atual sistema de cultivo de pimentas para consumo, a falta de práticas adequadas à cultura resulta em plantas com arquitetura desfavorável a captação de luz, consequentemente, há uma redução das taxas fotossintéticas e de ramos produtivos (redução do crescimento vegetativo). Associado a isto, as mudanças no padrão de distribuição de fotoassimilados resultam na redução de produtividade e ciclo produtivo pelo atraso no florescimento e, consequentemente, menor número de frutos na planta. Nesta condição, a poda surge como prática cultural visando modificar o crescimento das plantas e/ou aumentando a produção, além da melhoria da qualidade de frutos e também como forma de prevenção de doenças, por ocasião da retirada de ramos infectados.

A alteração na arquitetura espacial ou das dimensões de uma planta ocorrerá de acordo com o objetivo de exploração, seja para melhorar sua aparência ou funcionalidade, podendo elevar a produtividade e a qualidade de frutos. Na olericultura, a prática da poda foi estudada em algumas espécies, como o tomateiro (Almeida Guimarães et al., 2014; Oliveira et al., 2015); o pepino (Santi et al., 2013; Sediyama et al., 2014); a melancia (Casali et al., 1982); a abóbora e a abobrinha (Sonnenberg et al., 2007; Freitas et al., 2014), o melão (Vida et al., 2004) entre outras.

As pimenteiras em geral, possuem uma diversidade de forma e tamanho, e poucos estudos vem sendo conduzidos no sentido de melhorar, a partir de tratos culturais básicos, o crescimento e arquitetura geral das plantas para que possam ser cultivadas em vaso, de forma a se obter uma harmonia da planta com o vaso. A harmonia ou padrão de copa faz referência ao arranjo espacial dos órgãos de uma planta acima do nível do solo. Assim, este é um atributo de grande importância no cultivo de plantas ornamentais visando formar uma copa bem equilibrada. No entanto, o efeito buscado com a poda, será dependente da região de crescimento, da parte da planta que será podada, do tipo de poda e da época em que ela será realizada.

A auxina parece ser o hormônio responsável pelo controle da dominância apical. Este hormônio é produzido principalmente nas regiões apicais, movimentandose basipetamente para as outras regiões da planta. Quando há uma maior relação auxina/citocinina, a planta tende a formar mais brotos apicais. O contrário também é verdadeiro, ou seja, quando a relação auxina/citocinina diminui há uma tendência de formação de mais brotos laterais (Awad, 1983). Este efeito é então conseguido com algumas modalidades de poda. A retirada da gema apical, fonte da dominância apical, estimula a brotação das gemas axilares e consequentemente a ramificação lateral (Chen et al., 1997). Este é um aspecto importante, pois, corroborando com a idéia discutida anteriormente, de que a harmonia da copa é resultado do seu arranjo espacial, o grau de ramificação ou de brotações laterais nas plantas será um fator de extrema importância para alcançar este efeito visual.

Portanto, o presente estudo teve como objetivo avaliar o efeito da poda de formação no ciclo fenológico de pimenteiras ornamentais e comestíveis.

\section{MATERIAL E MÉTODOS}

O experimento foi desenvolvido na casa de vegetação do setor de floricultura e laboratório de pós-colheita do Departamento de Fitotecnia da Universidade Federal de Viçosa (UFV). Foram avaliadas duas cultivares comerciais Biquinho (Capsicum chinense) e Pirâmide Ornamental (Capsicum frutescens). As mudas de pimenteiras foram produzidas em ambiente protegido, em bandejas de poliestireno preenchidas com substrato comercial Bioplant ${ }^{\circledR}$. Quando atingiram de 2 a 3 pares de folhas foram transplantadas para vasos com capacidade de $900 \mathrm{~mL}$ ( $11 \mathrm{~cm}$ de altura, $9,5 \mathrm{~cm}$ de diâmetro basal, 13,5 cm diâmetro superior). No decorrer do 
experimento, foi efetuada fertirrigação com $150 \mathrm{~mL} /$ vaso/semana com solução nutritiva contendo 14,4; 1,$95 ; 12,92 ; 2,5 ; 1,0 ; 2,44 \mathrm{mmol} / \mathrm{L}$ de $\mathrm{N}, \mathrm{P}, \mathrm{K}, \mathrm{Ca}, \mathrm{Mg}$, $\mathrm{S}$ e $30,5,50,40,2$ e $0,1 \mu \mathrm{mol} / \mathrm{L}$ de B, Cu, Fe, Mn, Zn e Mo, respectivamente. A primeira poda foi realizada quando as plantas apresentavam em média 4 a 5 pares de folhas verdadeiras. Os cortes foram realizados logo após o primeiro par de folhas completamente expandidas, retirando-se o ápice das plantas. Quando as plantas iniciaram as brotações laterais e estas estavam com mais de $5 \mathrm{~cm}$, foi realizada a segunda poda (Figura 1). Nesta última, foi retirado o excesso de brotações laterais, mantendo apenas as brotações mais vigorosas e que fossem opostas e alternas de forma a se obter os seguintes tratamentos: plantas com duas hastes, plantas com três hastes e plantas sem poda. Em todas os casos, foi utilizada tesoura de poda, sanitizada a cada planta em solução de hipoclorido de sódio a 100 $\mathrm{ppm}$. Os tratos culturais foram realizados conforme recomendações técnicas para a cultura (Pinto et al., 2007).

Quando 50\% da população apresentavam pelo menos $30 \%$ dos frutos completamente amadurecidos, ou seja, no ponto ideal para a comercialização, determinado visualmente (frutos com o tamanho máximo de crescimento e formato típico de cada espécie, com a cor específica demandada pelo mercado e sem murcha), foi avaliada a seguinte característica morfoagronômica baseada na lista de descritores quantitativos e qualitativos sugerida pelo IPGRI (1995):
Precocidade: Expressa pelo número de dias compreendidos entre o plantio e a maturação completa de pelo menos $30 \%$ dos frutos (ponto ideal de comercialização). Os resultados foram expressos em dias.

O experimento foi montado no delineamento inteiramente casualizado, com três tratamentos (controle, dois e três ramos) e cinco repetições, totalizando 30 unidades experimentais. Cada unidade experimental foi constituída de um vaso.

\section{RESULTADOS E DISCUSSÃO}

O ponto ideal de comercialização, caracterizado pelo período compreendido entre o dia do transplantio até o dia em que a planta apresenta pelo menos $30 \%$ dos frutos maduros, foi retardado pela poda em $10 \mathrm{e}$ 15 dias para os genótipos Pirâmide Ornamental e Biquinho respectivamente (Tabela 1).

A fase vegetativa, período em que as plantas apresentaram desenvolvimento vigoroso sem vestígios de diferenciação para a fase de floração, aumentou consideravelmente quando as plantas foram submetidas

Tabela 1 - Precocidade dos genótipos Biquinho e Pirâmide Ornamental submetidos à poda

\begin{tabular}{lccc}
\hline \multirow{2}{*}{ Genótipos } & \multicolumn{3}{c}{ Precocidade } \\
\cline { 2 - 4 } & Sem poda & 2 hastes & 3 hastes \\
\hline Biquinho & 55 & 70 & 70 \\
Pirâmide ornamental & 60 & 70 & 70 \\
\hline
\end{tabular}
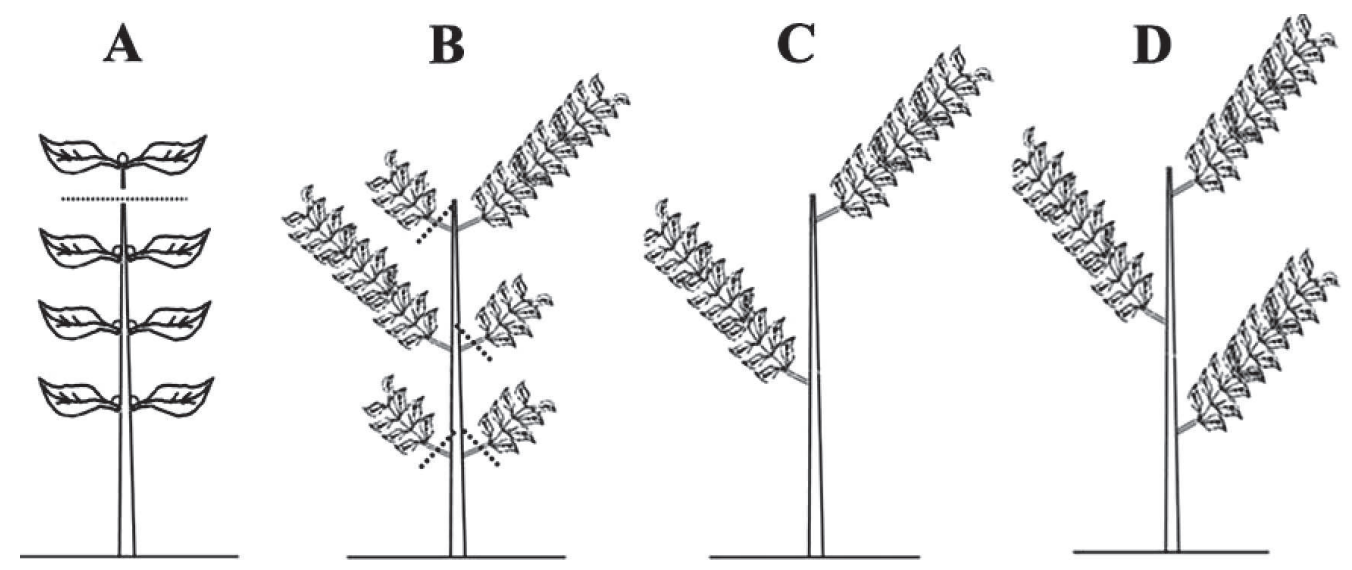

Figura 1 - Esquema da primeira poda de formação realizada nas pimenteiras. (A) Retirada da gema apical para estimular o crescimento dos ramos laterais, (B) Poda de seleção dos ramos laterais e (C) planta conduzidas com dois ramos e (D) plantas conduzidas com três ramos. 
à poda. No entanto, as fases de frutificação e maturação dos frutos não foram influenciadas pela poda. $\mathrm{O}$ ciclo fenológico das plantas podadas foi maior quando comparado com as plantas não podadas, isso ocorreu, provavelmente, pelo fato de que a poda fez com que as plantas precisassem de um período de tempo maior para recompor a copa.

Na cultivar Biquinho, foi observado ciclo fenológico para as plantas sem poda, com 2 hastes e 3 hastes de 80, 95 e 95 dias respectivamente. Já para a cultivar Pirâmide Ornamental o ciclo fenológico para as plantas sem poda, com 2 hastes e 3 hastes de 85, 95 e 95 dias respectivamente (Figura 2).

Valores semelhantes foram encontrados em experimento realizado por Backes et al. (2007) que avaliou substratos e doses de adubação na produção de pimenteira ornamental cultivar Gion Red, onde verificaram que o ciclo das plantas, ou seja, o intervalo de tempo compreendido entre o transplante até o ponto de colheita, foi de 56 dias. Esses dados, comparados com os valores citados por Sakata (2001), revelam uma redução do ciclo da cultura, provavelmente, relacionado com as condições climáticas, que são diferentes das regiões produtoras tradicionais de pimenta ornamental.

Vários fatores (genéticos e ambientais) podem influenciar as características e comportamento agronômico e fisiológico das pimenteiras, dentre eles o seu ciclo fenológico. E para que o cultivo e manejo sejam conduzidos de forma adequada, é essencial o entendimento da dinâmica de suas fases, sendo os estudos fenológicos uma ferramenta essencial para tal finalidade (d'Eça Neves \& Morellato, 2004). Além disso, permite inferir as respostas das plantas às diferentes condições climáticas e edáficas e das formas de cultivo (Fournier, 1974).
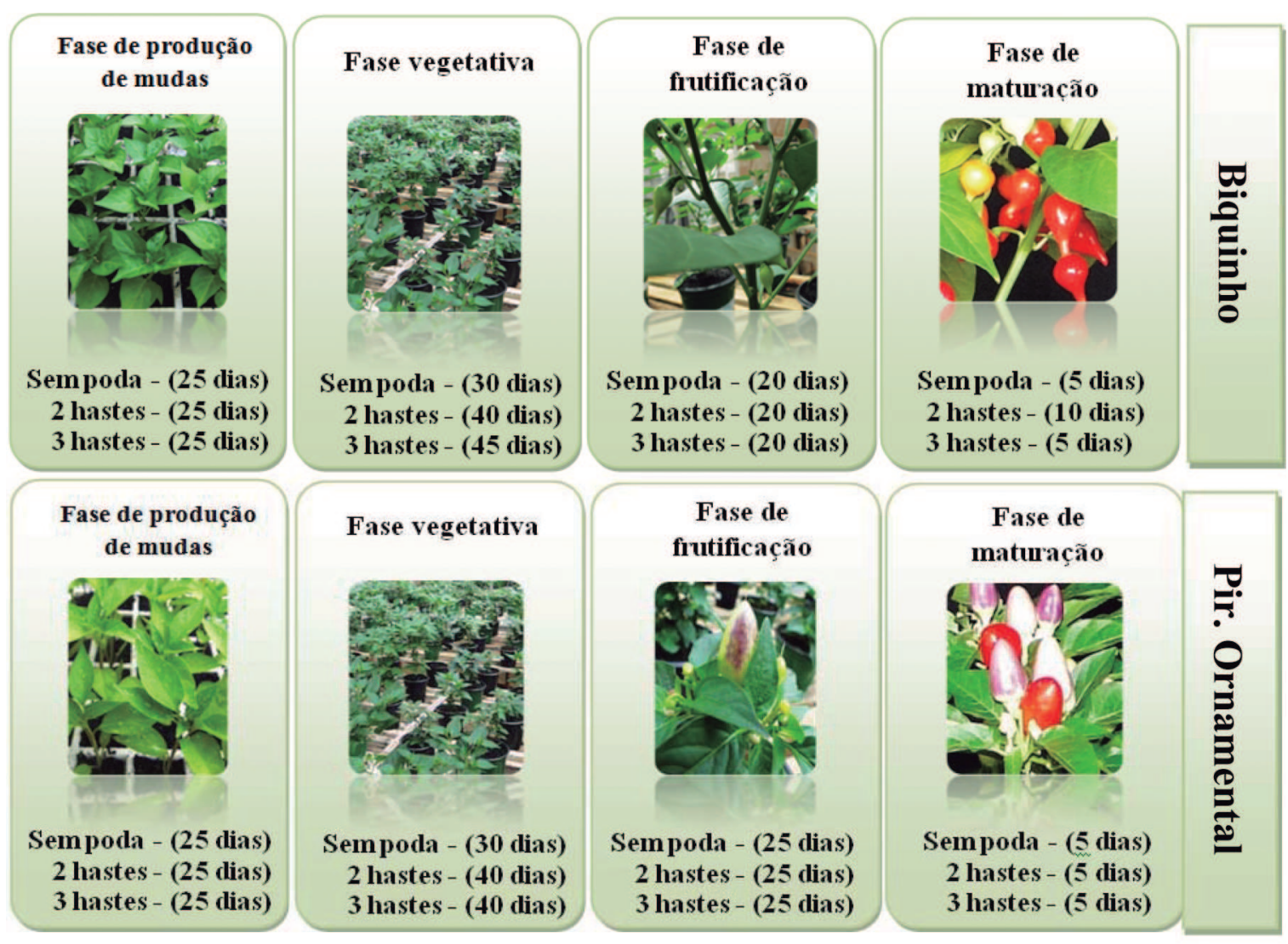

Figura 2 - Duração de cada fase durante o ciclo fenológico das pimenteiras. 
A caracterização fenológica das pimenteiras submetidas às condições de poda pode fornecer informações sobre prováveis datas de floração e frutificação (o que de fato interessa em pimenteiras ornamentais) e até mesmo a colheita, podendo, assim, indicar o potencial da poda como técnica a ser incorporada na produção de pimenteiras ornamentais em vasos.

\section{CONCLUSÕES}

A poda retardou em 15 e 10 dias a precocidade dos genótipos Biquinho e Pirâmide Ornamental, respectivamente. No entanto, a fase de frutificação e maturação não foram alteradas, com exceção do genótipo Biquinho conduzido em duas hastes que retardou em 5 duas a fase de maturação.

\section{REFERÊNCIAS BIBLIOGRÁFICAS}

ALMEIDA GUIMARÃES, M.; SILVA, D.J.H.; FONTES, P.C.R. et al. Produtividade e sabor dos frutos de tomate do grupo salada em função de podas. Bioscience Journal, v.24, n.1, p.32-38, 2008.

AWAD, M. Introdução a fisiologia vegetal. In: AWAD, M.; CASTRO, PRC. São Paulo: Nobel, 1983.

BACKES, C.; FERNANDES, F.M.; KROHN, N.G. et al. Produção de pimenta ornamental em função de substratos e doses de adubação com fertilizantes de liberação lenta e tradicional. Scientia

Agraria Paranaensis, v.6, n.1-2, p.67-76, 2007.

CASALLI, V.W.D.; SONNEMBERG, P.E.; PEDROSA, J.F. Melancia: cultivares e métodos culturais. Informe Agropecuário, v.8, p.2932, 1982.

CHEN, J.G.; ZHAO, H.Y.; ZHOU, X. et al. Flutuation in levels of endogenous hormones after decapitation and 6-benzyl amino purine treatement in azalea, and their relationship to apical dominance. Scientia Horticulturae, v.17, p.49-58, 1997.

CRISÓSTOMO, J.R. et al. Cultivo de pimenta tabasco no Ceará. Fortaleza: Embrapa Agroindústria Tropical, 2006. 34p.
DALASTRA, I.M.; PIO, R.; CAMPAGNOLO, M.A. et al. Épocas de poda na produção de figos verdes' Roxo de Valinhos' em sistema orgânico na região oeste do Paraná. Revista

Brasileira de Fruticultura, v. 31, n.2, p.447-453, 2009.

d'EÇA NEVES, F.F.; MORELLATO, P.C. Métodos de amostragem e avaliação utilizados em estudos fenológicos de florestas tropicais. Acta

Botanica Brasilica, v. 18, n.1, p.99-108,2004.

FOURNIER, L.A. Un método cuantitativo para la medición de características fenológicas en árboles. Turrialba, v.24, n.4, p.422-3,1974.

FREITAS, P.G.N.; CLÁUDIO, M.T.R.; TAVARES, A.E.B. et al. Poda apical para produção de frutos e sementes de abóbora.Agro@mbiente Online, v.8, n.2, p.230-237, 2014.

INTERNATIONAL PLANT GENETIC RESOURCES INSTITUTE. IPGRI. Descriptors for Capsicum. Rome: IBPGR, 1995. 49p.

NUNES, L.; PATRÍCIO, M.S.; CORTES, P. Manutenção de árvores. In: AZEVEDO, J.C.; GONÇALVES, A. (Coords.) Manual de boas práticas em espaços verdes. Bragança: Câmara Municipal de Bragança, 2010. p.111-117.

OLIVEIRA, V.R.; FONTES, P.C.R.; CAMPOS, J.P. et al. Qualidade do tomate afetada pelo número de ramos por planta e pela poda apical. Ceres, v.43, n.247, 2015.

PINTO, C.M.F.; CRUZ, R.M. Agronegócio pimenta em Minas Gerais. In: CONGRESSO BRASILEIRO DE OLERICULTURA, 51.

Horticultura Brasileira 29. Viçosa: $\mathrm{ABH}$, S5744-S5765, 2011.

SANTI, A.; SCARAMUZZA, W.L.M.P.; SOARES, D.M.J. et al. Desempenho e orientação do crescimento do pepino japonês em ambiente protegido. Horticultura Brasileira, v.31, n.4, p.649-653, 2013.

SAKATA. Flower seed catalogue. Bragança Paulista: Sakata sementes, p.15, 2001.

SARLI, A.E. Horticultura. Buenos Aires: Editorial ACME, 1958. 454p. 
SEDIYAMA, M.A.N.; NASCIMENTO, J.L.M.; LOPES, I.P.C. et al. Influência de poda das plantas na produtividade de frutos de pepino dos grupos Aodai, Japonês e Caipira. Horticultura Brasileira, v.32, n.04, p.491-496, 2014.

SOARES, J.A.; KOLLER, O.C. Estudo preliminar sobre sistemas de poda em tomateiro. Revista de Olericultura, v.4, p.131-134, 1964.
SONNENBERG, P.E.; BORGES, J.D.; DUARTE, J.B. Efeitos de poda de rama na produção de abobrinha (Curcubita moschata) cv."menina brasileira". Pesquisa Agropecuária Tropical, v.24, n.1, p.123-127, 2007.

VIDA, J.B.; TESSMAN, D.J.; ZAMBOLIM, L. et al. Controle da podridão gomosa em melão rendilhado em cultivo protegido por sanitização de ferramenta de poda. Fitopatologia Brasileira, v.29, p.626-630, 2004.

Recebido para publicação em 11/3/2017 e aprovado em 19/5/2017. 